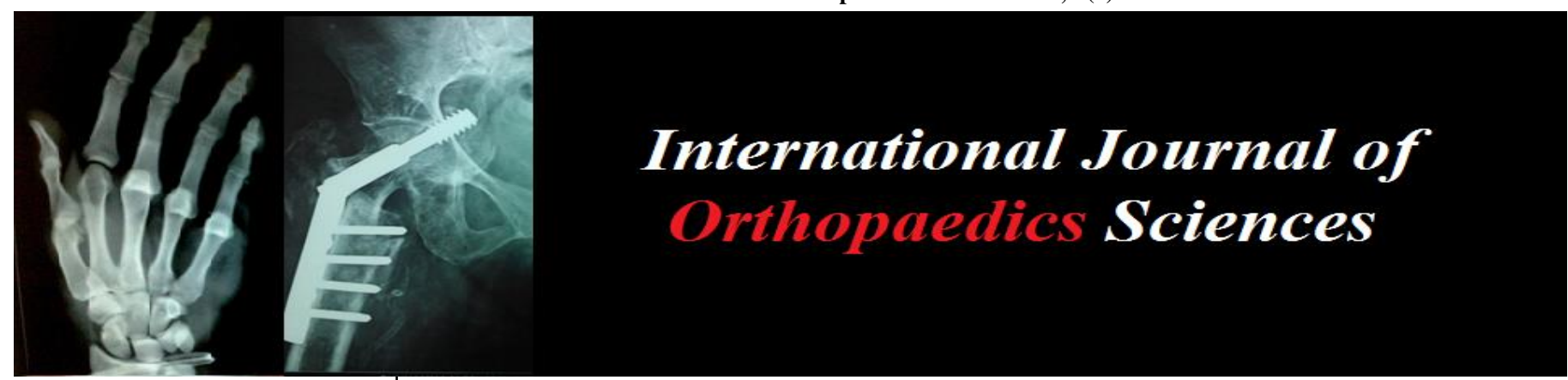

E-ISSN: 2395-1958

P-ISSN: 2706-6630

IJOS 2020; 6(1): 455-459

(C) 2020 IJOS

www.orthopaper.com

Received: 24-11-2019

Accepted: 28-12-2019

Nawfal N

Vydehi Institute of Medical

Sciences \& Research Centre,

Bengaluru, Karnataka, India

\section{Karthik MN}

Vydehi Institute of Medical Sciences \& Research Centre, Bengaluru, Karnataka, India

Satish Kumar C

Vydehi Institute of Medical Sciences \& Research Centre, Bengaluru, Karnataka, India

Corresponding Author: Karthik MN

Vydehi Institute of Medical Sciences \& Research Centre, Bengaluru, Karnataka, India

\section{Reverse hybrid total hip arthroplasty, a good alternative to uncemented total hip arthroplasty}

\author{
Nawfal N, Karthik MN and Satish Kumar C
}

DOI: https://doi.org/10.22271/ortho.2020.v6.i1h.1906

\section{Abstract}

Total hip arthroplasty is the treatment of choice for primary and secondary arthritis of the hip joint. Uncemented total hip is the implant of choice in young adults with good bone stock in the acetabulum and femur. Correspondingly, cemented implants are preferred in patients with poor bone quality and in elderly. Uncemented prosthesis are relatively expensive when compared to cemented but have longer viability. A prospective study conducted at Vydehi institute of medical sciences between July 2016 and may 2018 on 21 patients using reverse hybrid total hip arthroplasty and follow up was done post op, 6 , 12, 24 weeks and 1 year post operatively. Functional outcome assessed using Modified Harris Hip score. The data was analyzed using a Chi square test for equal proportions was applied for categorical variables and Independent sample t test was applied. The mean pain score during pre-operative period was $11.43 \pm 3.59$ and during post-operative period was $41.71 \pm 4.26$. The difference between the pre and postoperative pain scores was statistically significant. The mean functional gait score during pre-operative stage was $10.24 \pm 8.23 \&$ during post-operative stage was $41.71 \pm 4.26$, the $t$ value corresponding to this mean difference was -8.609 and its corresponding $p$ value was $0.000<0.05$. Since $p$ value is less than 0.05 , there was statistically significant difference between the pre and post-operative scores. The mean functional activity score during pre-operative stage was $5.29 \pm 2.47$ and during post-operative stage was $12.0 \pm 1.41$. There was a statistically significant difference between the pre and post-operative scores in the study group. The mean total score during pre-operative stage was $33.43 \pm 12.33$ and during postoperative stage was $89.43 \pm 8.73$. The difference between pre and post-operative scores was statistically significant.

Keywords: Prospective study, reverse hybrid, total hip arthroplasty, modified harris hip score

\section{Introduction}

In cemented THR, the stem and the acetabular cup are held in place with acrylic bone cement but cement less prosthetic components use the tensile elasticity of the bone as a method of fixation. There are two reasons proponents of un-cemented prosthesis favour the procedure. First, eventually all THR will fail, usually after more than 10 years. When they fail, loosening of the stem is amongst the most prevalent causes. The quality of fixation of cemented stem tends to degrade over time while cement less stems on the other hand, are press-fitted into the femoral canal, causing expansion of it. This introduces high tensile stresses on the femur that hold the stem in place. With time, cement less stems induce bone growth and make permanent bond with bone, which is the main cause for why cement less stems often perform better on the long-term than cemented stems. Secondly, when the stems fail, they have to be replaced. Removing cement less stems is very easy in comparison with cemented stems. When the bone cement is removed, some amount of bone tissue can follow, further weakening a weak bone but also, the stem-bone interface is less capable of receiving another implant. However, not all femurs can handle the press-fitting surgery of implanting cement less stems; the bone has to be strong enough. This has to be examined before surgery to choose the correct implant. The hip joint is a load-bearing joint, which is constantly receiving high loads. These loads lead to gradual degradation of articular surfaces, causing functional impairment and pain ${ }^{[1]}$.

Cemented femoral component provides an immediate postoperative advantage in terms of better integration between bone, cement and the prosthesis, which permits dramatic early relief of pain and early weight-bearing. In the short term outcome, studies show almost all the relevant studies reported superiority of the cemented fixation to the un-cemented in terms of 
pain reduction. Therefore, it can be concluded that for the clinical and functional outcome, the cemented group tends to be equal, if not superior, to the un-cemented group ${ }^{[2]}$.

Surgeons noted an increased rate of acetabular loosening relative to femoral loosening in cemented arthroplasties, the concept of the "hybrid" total hip arthroplasty has been adopted by many surgeons ${ }^{[3]}$.

Cementing the stem using contemporary techniques allows earlier unrestricted weight bearing and yields a lower incidence of low grade thigh pain. Leaving the acetabular component uncemented avoids the consequences of cement fragmentation and loosening. In many centres, hybrid total hip arthroplasty is now the preferred technique for primary hip arthroplasty in patients older than 60. Results in patients observed for two to four years show that the hybrid arthroplasty performs as well as cemented total hip arthroplasty in the short term ${ }^{[3]}$. But again problems like aseptic loosening of femoral stem came up which made the surgeons again think of an alternative option. Henceforth the idea of reverse hybrid total hip replacement evolved.

Reverse Hybrid Total Hip Arthroplasty is a procedure where in cemented all poly acetabular cup and uncemented
Hydroxyapatite coated femoral stem with head is used for total hip arthroplasty

\section{Methodology}

A prospective study was conducted on patients who underwent Reverse Hybrid THR for primary hip replacement in Department of Orthopaedics, Vydehi Institute of Medical Sciences And Research Centre, Whitefield, Bangalore between July 2016 to May 2018. A sample size of 21 Patients was obtained. The clearance from institutional ethical committee was obtained before starting the study. Informed, Bilingual and Written consent was taken from the patients included in the study. Patients with hip pain was admitted and examined according to protocol both clinically and radiologically, and functional outcome is assessed by distribution of Modified Harris hip scores both preoperatively and post operatively. And the patients were reviewed with post op X-Rays immediately after surgery at the end of 3,6 months, 1year \& annually thereafter.

\section{Results}

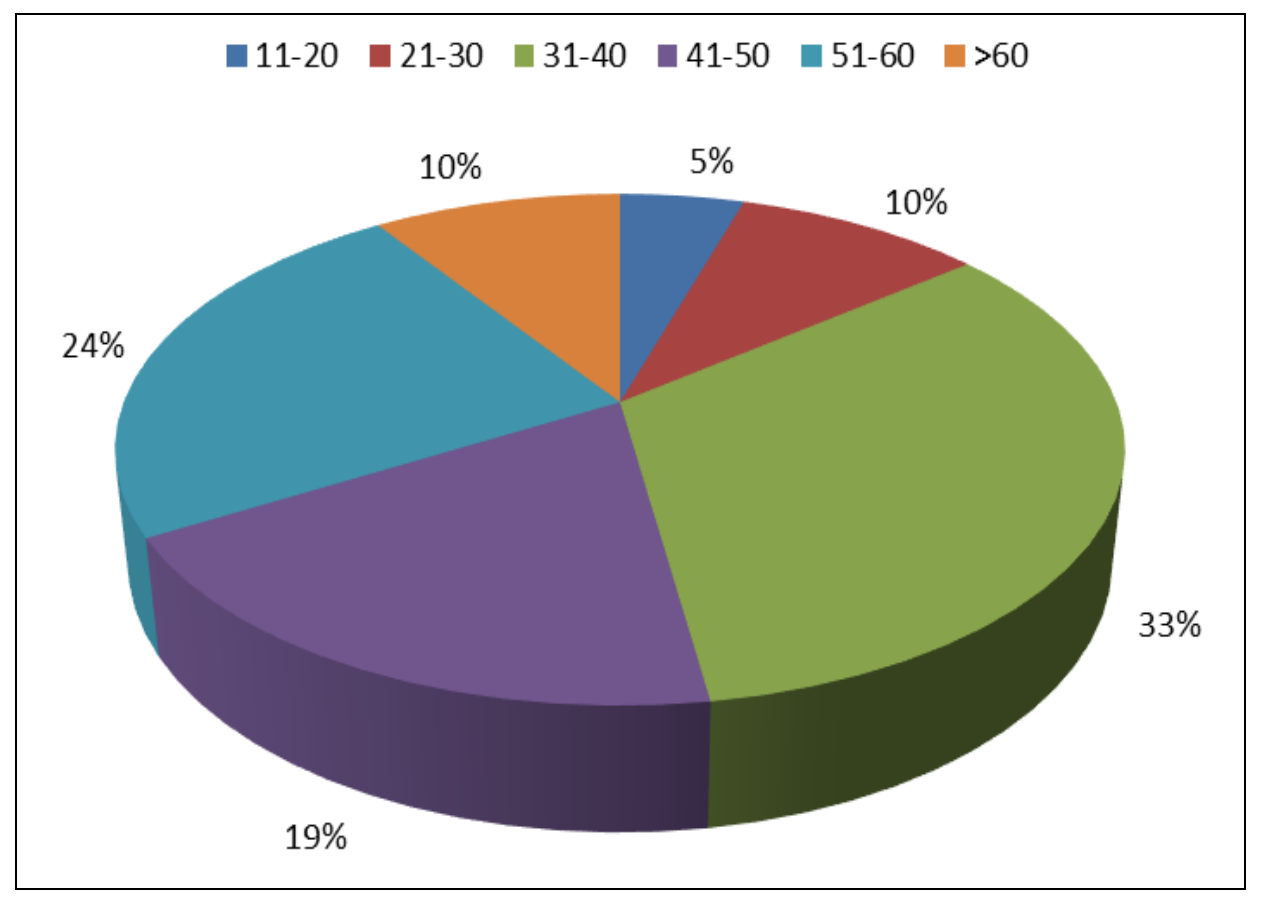

Fig 1: Age Distribution in the study group

Table 1: Etiology for THR in the study group

\begin{tabular}{|c|c|c|}
\hline Etiology & No. of patients & \% \\
\hline Non-union \# neck of femur & 4 & $19 \%$ \\
\hline Steroid induced AVN & 3 & $14 \%$ \\
\hline Secondary osteoarthritis hip & 9 & $43 \%$ \\
\hline Failed Osteosynthesis \# neck of femur & 1 & $5 \%$ \\
\hline Idiopathic AVN & 4 & $19 \%$ \\
\hline Total & 21 & 100.0 \\
\hline
\end{tabular}

Table 2: Total Score in the study group

\begin{tabular}{|c|c|c|c|c|c|}
\hline Total Score & Mean & SD & Minimum & Maximum & $\begin{array}{c}\text { t value } \\
\text { p value }\end{array}$ \\
\hline Pre-Operative & 33.43 & 12.33 & 17 & 61 & -17.802 \\
\hline Post-Operative & 89.43 & 8.73 & 63 & 99 & $\begin{array}{c}0.000, \\
\text { Significant }\end{array}$ \\
\hline
\end{tabular}

\section{Complications}

Table 3: Complications of the study group

\begin{tabular}{|c|c|c|}
\hline Complications & No of patients & Percent \\
\hline Superficial infection & 3 & 14 \\
\hline Nerve injury & 1 & 5 \\
\hline Peri prosthetic Fracture & 1 & 5 \\
\hline Limb Length Discrepancy & 1 & 5 \\
\hline
\end{tabular}

Case 1 


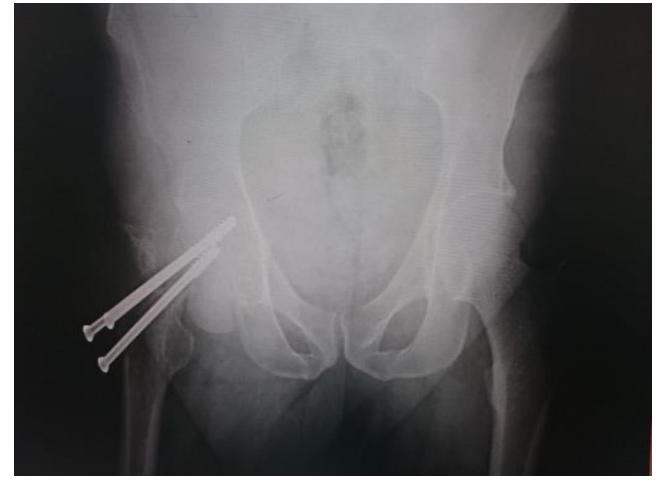

Fig 1a: Pre op X Ray

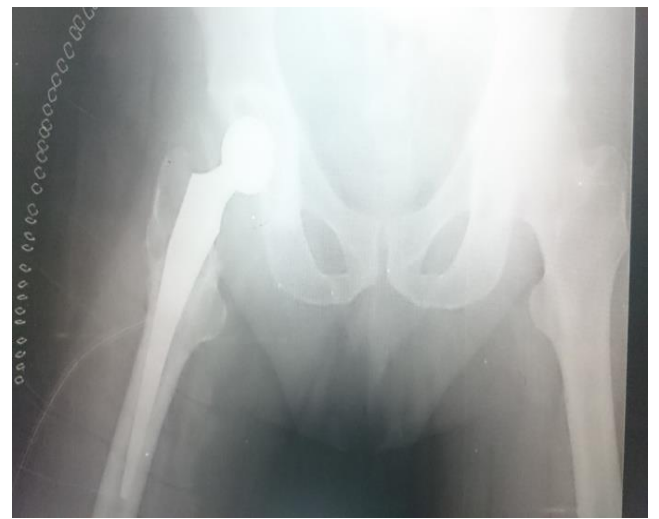

Fig 1b: Post Op Day 1

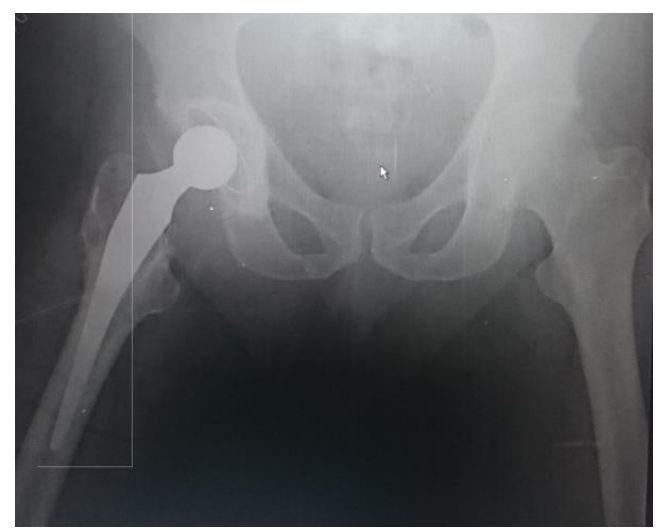

Fig 1c: Post Op 6 Months

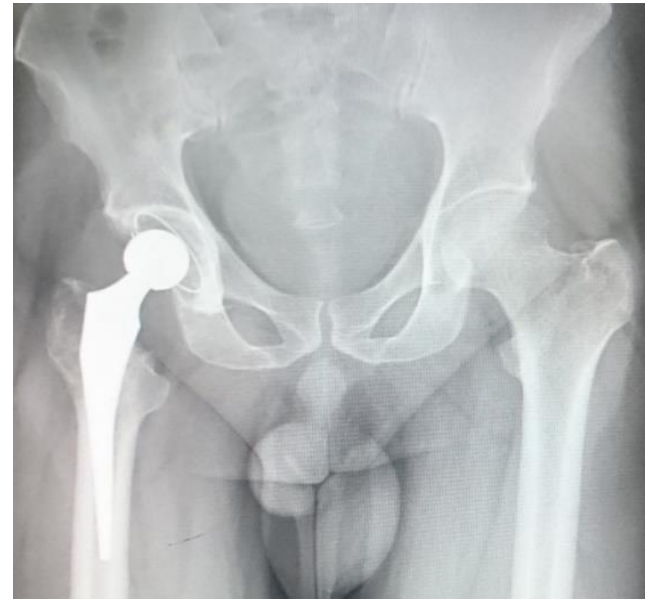

Fig 1d: Post Op 2 Years

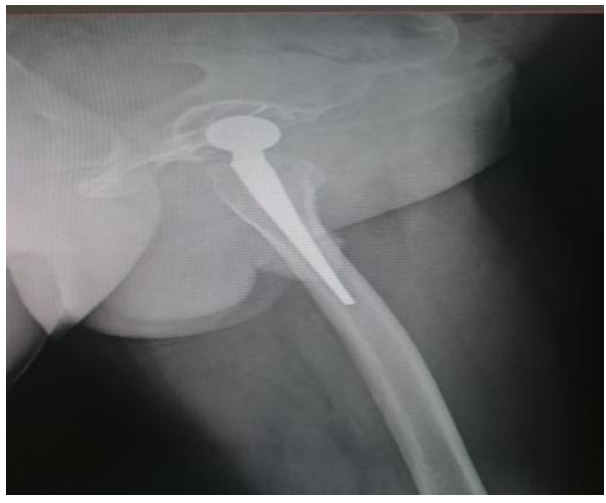

Fig 1e: Post Op 2 Weeks

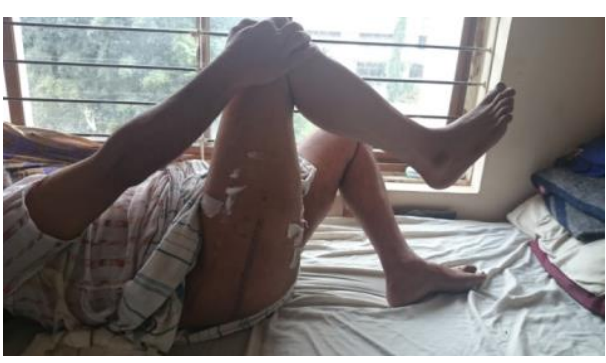

Fig 1f: Flexion

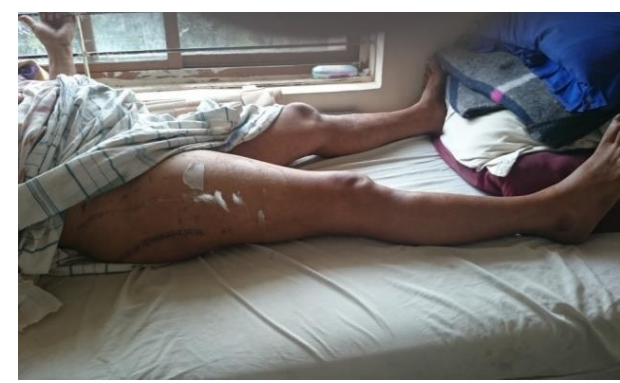

Fig 1g: Extension

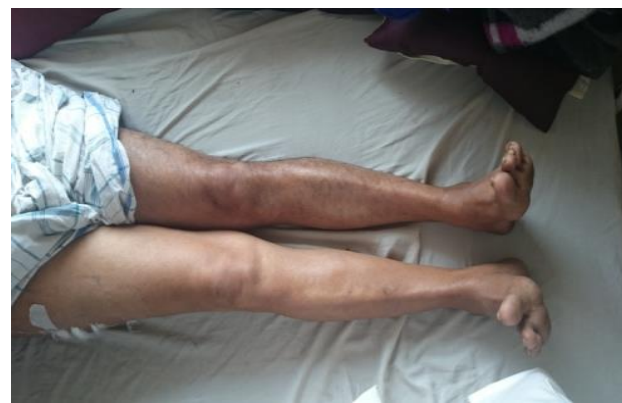

Fig 1h: Limb Measurement

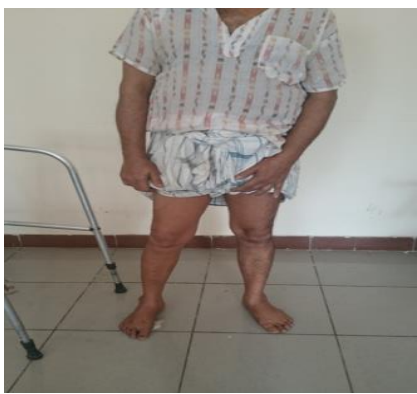

Fig 1i: Weight Bearing 


\section{Post Op 3 Months}

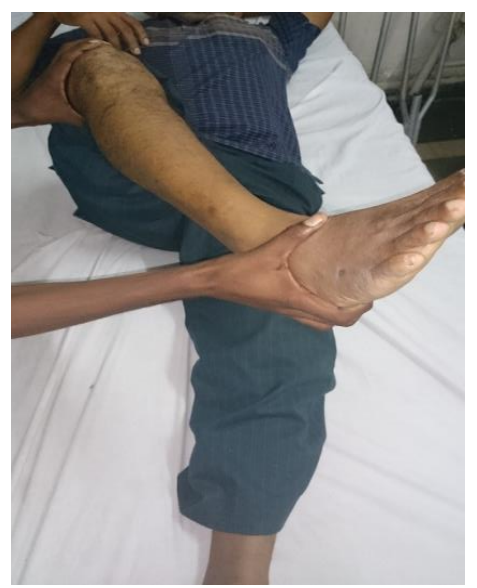

Fig 1 j: Internal Rotation

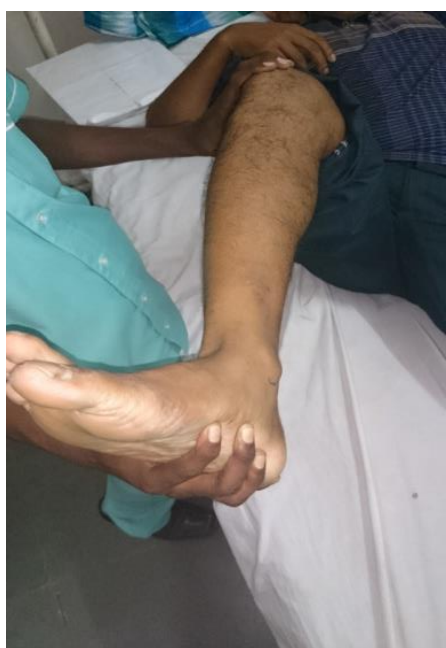

Fig 1k: External Rotation

Case 2.

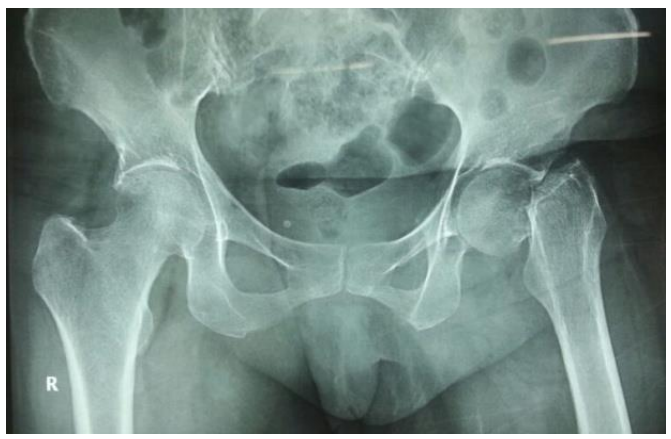

Fig 2a: Pre-Op Xray

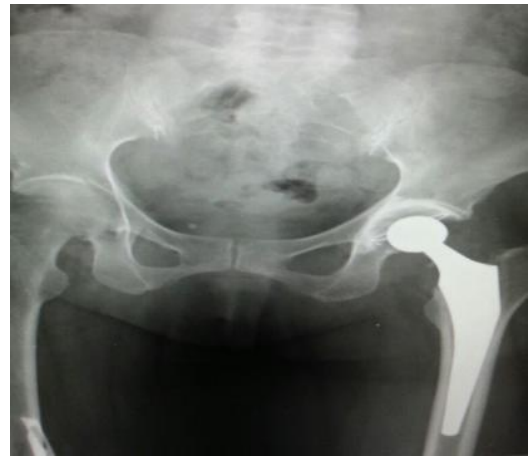

Fig 2b: Post Op Day 1

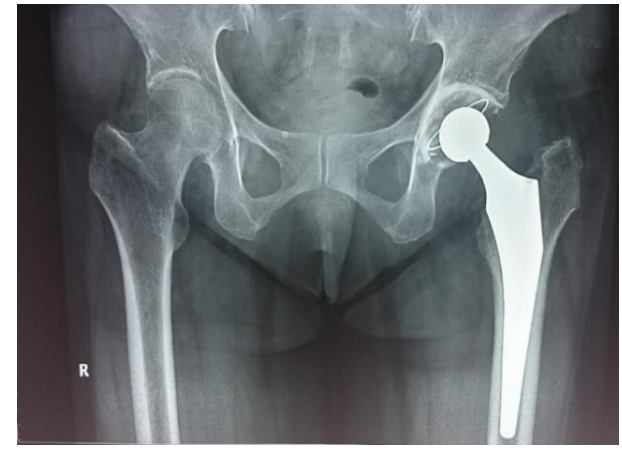

Fig 2c: Post Op 3 Months

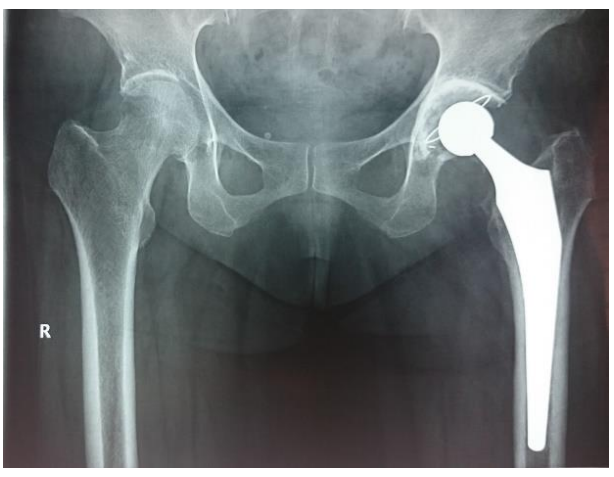

Fig 2d: Post Op 6 Months

\section{Post Op After 1 Month}

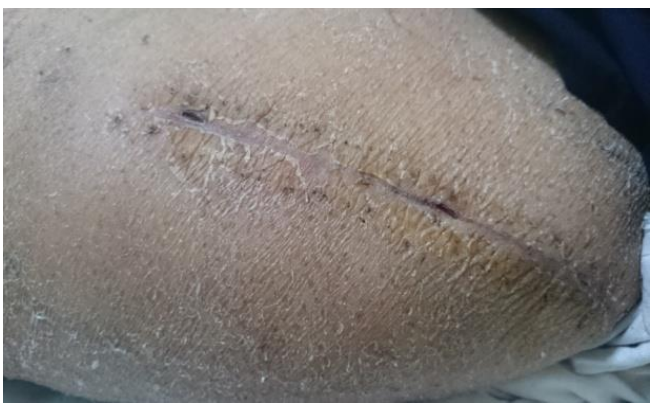

Fig 2e: Post Op Scar

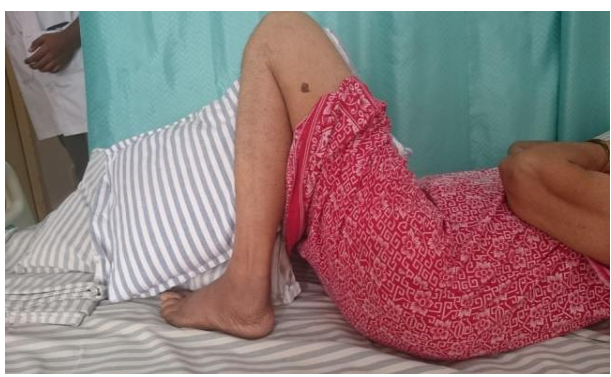

Fig 2f: Flexion

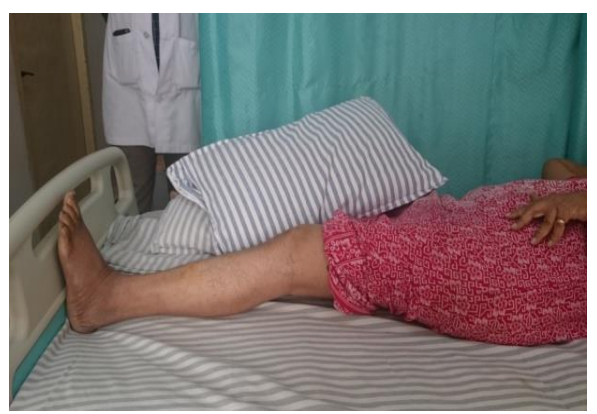

Fig 2g: Extension 


\section{Discussion}

The mean age of patients in the study group was 35.95 \pm 14.62. About $33 \%$ of the patients in the study group belonged to 31-40 years age group. It was followed by patients of age group 51-60 (24\%), 41-50(19\%), 21-30(10\%), >60(10\%) and less than $20(5 \%)$. The gender distribution of the study group constitutes $67 \%$ of males and $33 \%$ of females. Other studies done by Merrill A. Ritter, MD, Matthew R. Galley et al. \& Lindalen E, Havelin LI et al. also found the same results ${ }^{[4,5]}$ In our study our consideration was about bone quality for implant fixation. We had not set an age limit for the surgery $\&$ we strongly believe that age is not a criteria for this type of arthroplasty. This view was supported by our results.

\section{Etiology}

In the study group etiology for THR in $43 \%$ of patients constitutes of Secondary osteoarthritis hip. $19 \%$ each of the group had Idiopathic avascular necrosis \& Non union \# neck of femur. $14 \%$ of the group had steroid induced AVN followed by $5 \%$ of patients with Failed osteosynthesis \#Neck of Femur. Two Indian authors have done Reverse Hybrid THR for Neglected antero inferior dislocation of Hip \& Osteoarthritis Hip respectively ${ }^{[8,9]}$ Lindalin. E, Hanelin. L.J et al also have done Reverse Hybrid THR in Secondary Osteoarthritis \& AVN Hip ${ }^{[5]}$. Maruyama et al has included eighty patients with secondary osteoarthritis hip in their comparative study of Cemented vs Reverse hybrid THR ${ }^{[7]}$.

The Harris Hip score of $90.5 \%$ of the study group was 4 and $9.5 \%$ of the patients was 0 during pre operative stage. All the patients had a score of 4 in the study group post operatively. The mean total score during pre operative stage was $33.43 \pm 12.33$ and during post operative stage was $89.43 \pm 8.73$. This score is comparable to the scores achieved by SM Lan, Kuo-An Lai et al. ${ }^{[6]}$

Most $(71 \%)$ of the patients in the study group had no complications. The common complication in the study group was Superficial infection in 3 patients which is $14 \%$. One patient each had limb length discrepancy and peri-prosthetic fracture. In a study by SM Lan et al no loosening, migration or progressive radiolucent lines around the cup was noted in any patient. One patient had Vancouver type 2B periprosthetic fracture ${ }^{[6]}$. Ritter et al study also had a case of periprosthetic fracture. They further observed that no patients in the reverse hybrid group reported significant post operative complications including polyethylene wear, osteolysis, or evidence of acetabular aseptic loosening ${ }^{[4]}$. Lindalen et al observed there is no statistical differences between cemented and reverse hybrid THRs in case of deep infection, dislocation and aseptic loosening of stem ${ }^{[5]}$. Superficial infections in our patients were dealt with antibiotics and regular dressings with betadine.

The condition of $90.5 \%$ of patients was very poor before operation and $9.5 \%$ patients had poor condition. The outcome after total hip replacement was excellent in $71.4 \%$ of the study group. About $14.3 \%$ of the study group had good outcome, $9.5 \%$ had fair results and one patient had poor outcome. We had poor result in one patient as rehabilitation was difficult in this patient. The patient sustained previous fractures to right distal $1 / 3^{\text {rd }}$ femur and left proximal $1 / 3^{\text {rd }}$ tibia one year ago prior to the surgery, for which open reduction and internal fixation was done else where. Patient had post op atrophy of the quadriceps muscles and restricted range of movements bilateral knee. These co-morbidities hampered our hip arthroplasty rehabilitation protocol in the patient. In studies by most of the proponents of Reverse Hybrid Total Hip
Arthroplasty and their outcomes were graded good to excellent in all the literature in comparison to the conventional cemented/ un-cemented THRs ${ }^{[4-6]}$.

\section{Conclusion}

This study suggests that the current concept of Reverse Hybrid Total Hip Arthroplasty used in total hip replacement for ailments of the hip joint, provides satisfactory clinical and radiographic outcomes after an intermediate duration follow up. Reverse Hybrid THR, although unusual, is a successful alternative to Uncemented THR when a need arises and institutional resources allow. Even though the procedure is not free of complications, the overall functional and clinical outcome had shown good to excellent result. So Reverse Hybrid THR can be considered as an alternative to the conventional arthroplasty procedures.

Declaration: The authors did not receive any funding for this research and there is no conflict of interest.

\section{References}

1. Pröstur Pétursson, Benedikt Magnússon, Benedikt Helgason, Gigja Magnúsdóttir, Grétar Halldórsson, Jan Tribel et al. Paolo Gargiulo Bone and muscle assessment in patients undergoing total hip arthroplasty using HU based analysis European Journal Translational Myology Basic Applied Myology. 2012; 22(3):147-152.

2. Ali Abdulkarim, Prasad Ellanti, Nicola Motterlini, Tom Fahey, John M. O'Byrne Cemented versus uncemented fixation in total hip replacement: a systematic review and meta-analysis of randomized controlled trials Orthop Rev (Pavia). 2013; 5(1):e8.

3. Harris WH. Hybrid total hip arthroplasty. Clin Orthop, 1989; 249:21-29.

4. Ritter MA, Galley MR. Acetabular Fixation Options in Total Hip Replacements, Healio Orthopaedics, September 2008; 31(9)

5. Lindalin E, Hanelin LJ, Nordsletten L, Dybaik E. Is Reverse Hybrid Hip Replacement The Solution?, Acta Orthopaedics. 2011; 82(6):639-645.

6. Lan SM, Lai KA. Reverse Hybrid Total Hip Arthroplasty in select patients, Formosan Journal of Musculoskeletal Disorders. 2010; 1(1):20-25.

7. Masaaki Maruyama, Shinji Wakabayashi, Keiji Tensho. Reverse Hybrid Total Hip Arthroplasty: Comparative Study With All-Cemented Total Hip Arthroplasty Bone Joint J. 2013; 95-B(15):254.

8. Satish M Bobade, Anirudha Page. Total hip arthroplasty for hip arthritis with protrusio acetabuli using autograft reconstruction and metal wire mesh Med J DY Patil Univ. 2013; 6:428-31.

9. Dr. Kiran S Patil, Dr. Deepesh Daulatni, Dr. Monish Bami, Dr. Mallikarjun Ithli. Neglected Antero-Inferior Dislocation of Hip Treated With Primary Reverse Hybrid THR-A Rare Case Report. World Journal of Medical and Surgical Case Reports. 2014, 4. 